\title{
Kikuchi-Fujimoto necrotizing lymphadenitis: A rare case of generalized lymphadenopathy
}

\author{
Charlotte Lansky, Hasaam Sheikh, Mohamad Lazkani* and Cheryl O'Malley \\ Department of Internal Medicine, Banner University Medical Center, Phoenix, Arizona, USA
}

\begin{abstract}
Introduction: Kikuchi-Fujimoto Disease, or histiocytic necrotizing lymphadenitis, is a rare condition with a benign course that mimics malignancy on presentation. Careful examination and histologic confirmation of the diagnosis is therefore critical.

Case presentation: A 53-year old female who presented with fever, generalized lymphadenopathy, and constitutional symptoms underwent lymph node biopsy after concern for malignant lymphoma.
\end{abstract}

Management and outcome: The patient was diagnosed with Kikuchi-Fujimoto Disease after histopathologic confirmation of necrosis with abundant histiocytes and absent neutrophils.

Discussion: Fever and lymphadenopathy carries a broad differential, and a missed diagnosis of a rare condition like Kikuchi-Fujimoto disease can lead to inappropriate treatment in an otherwise benign condition.

\section{Introduction}

Kikuchi-Fujimoto Disease (KFD), or Kikuchi histiocytic necrotizing lymphadenitis, is a benign, self-limited condition typically characterized by fever and focal lymphadenopathy. KFD has a worldwide distribution and affects both genders with a higher prevalence in young women and Asiatic people [1]. The true incidence is not known. The etiology of KFD is still debated in the literature, however infectious agents have been implicated, and more recent studies are suggestive of a viral etiology based on the associated histology and clinicopathology [2]. Although an association with systemic lupus erythematosous (SLE) has been debated throughout the literature, no definitive relationship has been proven. Interestingly, SLE must still be ruled out prior to confirming a diagnosis. KFD can presents with symptoms that typically last one to four months, yet recurrence

has been reported in 3-4\% of affected individuals [1]. We present a case of KFD in a woman with fever, generalized lymphadenopathy, and systemic symptoms that were diagnosed by lymph node biopsy, which was initially ordered based on a suspicion for malignant lymphoma.

\section{Case presentation}

A 53-year old African American woman presented with a twoweek history of fever, night sweats, fatigue, unintentional weight loss, and painful lymphadenopathy in the neck. She admitted a sixmonth history of similarly painful lymphadenopathy in the axilla. Her history was significant for headaches as well as chronic back pain selfmedicated with marijuana. An HIV antigen screen from one-year prior was negative.

Physical exam revealed tender, palpable lymph nodes in the posterior cervical chain, auricular area, axilla, and femoral area. The largest node was found in the right posterior cervical chain and measured $1.43 \mathrm{~cm}$ by ultrasound (Figure 1).
Initial lab results revealed a white blood cell (WBC) count of 3,000 composed of $50 \%$ lymphocytes, an erythrocyte sedimentation rate (ESR) of 105, and C-reactive protein (CRP) of 32.3. Baseline ESR and CRP obtained from the previous year were 10 and 1.6, respectively. ANA, lupus anticoagulant, and anti-smith antibodies were all negative. A lumbar puncture was notable for CSF with a WBC of 12 and protein of 47. EBV antigen and IgG was positive as well as Varicella IgG. CSF cultures and acid-fast smear were negative, as well as tissue, blood, and fungal cultures. Syphilis screening, CMV PCR, and serology for cryptococcal antigen, coccidioides, and toxoplasmosis were also negative. Contrast CT of the neck and chest demonstrated a $5 \mathrm{~mm}$ pulmonary nodule, no enlarged lymph nodes, and no evidence of acute

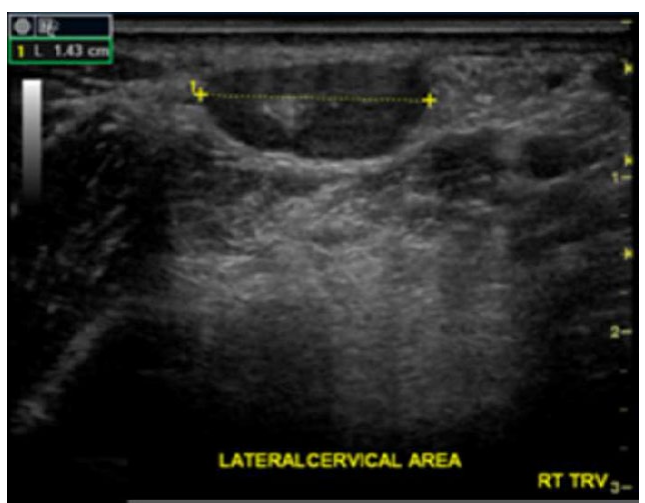

Figure 1. $1.43 \mathrm{~cm}$ lymph node in right posterior cervical chain on ultrasound.

Correspondence to: Mohamad Lazkani, Department of Internal Medicine, Banner University Medical Center, Phoenix, Arizona, USA, E-mail: mohamadlazkani@gmail.com

Received: April 20, 2016; Accepted: May 04, 2016; Published: May 09, 2016 
pulmonary embolism.

The patient's constitutional symptoms were initially concerning for a new diagnosis of malignant lymphoma, and an ultrasound-guided core biopsy and FNA of a lymph node in the right posterior cervical triangle was performed. Biopsy demonstrated sections of lymph node with focal areas of necrosis within the cortex characterized by fibrinoid material containing numerous small nuclear fragments (Figure 2). Within and surrounding these foci was a population of histiocytes and transformed lymphoid cells. Plasma cells were focally present, but neutrophils and granulomatous inflammation were absent (Figure 3). Immunohistochemical staining revealed CD68 positive histiocytes with few CD20 positive B lymphocytes in the necrotic areas and many larger, CD3 positive cells consistent with T cell origin [3].

The patient was diagnosed with KFD based on the histogologcial findings of necrotizing lymphadenitis, CD68 positive histiocytes, necrosis in the absence of neutrophils, and an abundance of histiocytes. The remainder of her hospital course was uneventful and she was subsequently discharged on glucocorticoid therapy with follow up by rheumatology.

\section{Discussion}

Kikuchi-Fujimoto Disease, or histiocytic necrotizing lymphadenitis, was first described in young women in Japan in 1972 and has since been a subject of study throughout the literature. It is characterized by fever and lymphadenopathy, yet a variety of associated signs and symptoms may be present, including systemic symptoms such as fatigue, weight loss, night sweats, and other less common manifestations such as

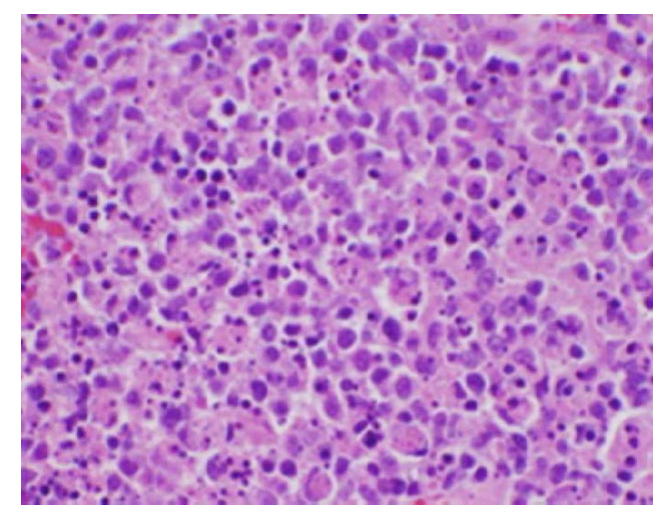

Figure 2. H\&E stain of lymph node biopsy demonstrating necrosis and absent neutrophils.

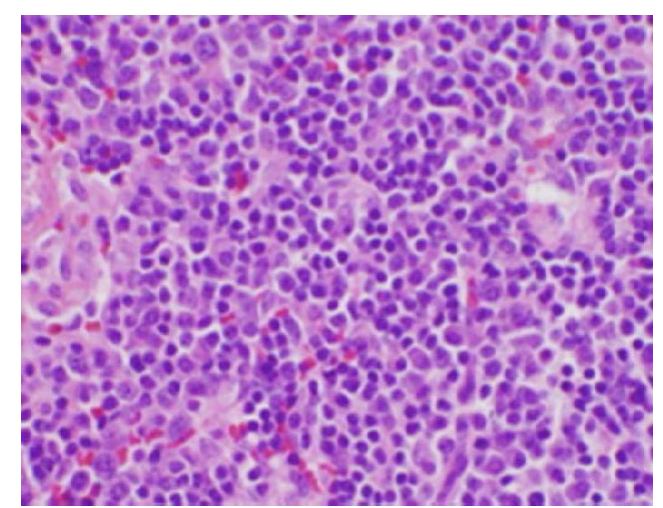

Figure 3. H\&E stain of cervical lymph node biopsy showing numerous histiocytes and absent neutrophils. aseptic meningitis, splenomegaly, rash, and arthralgias [4]. KFD most commonly presents as fever and unilateral cervical lymphadenopathy in young women, however the lymphadenopathy can also be multifocal. Extranodal involvement of the skin and bone marrow, for example, is often accompanied by systemic symptoms, including night sweats, weight loss, fatigue, nausea, vomiting, and diarrhea [4].

The fever and lymphadenopathy seen in KFD carries a differential diagnosis that is quite broad and includes infectious, autoimmune, and malignant etiologies. The differential diagnosis for fever and lymphadenopathy is diverse and encompasses a breadth of diseases with varying ranges of prognoses. Misdiagnosing a patient with a condition that has an unfavorable prognosis and, in particular, a more aggressive treatment regimen can lead to inappropriate care and poor medical consequences. Multiple reports have demonstrated that the symptomatology of KFD has been confused with conditions like tuberculosis and malignant lymphoma [4,5]. As such, it is important for clinicians to be aware of and include Kikuchi-Fujimoto Disease, albeit a rare condition with a variable presentation, within the differential for fever and lymphadenopathy.

The exact pathogenesis remains uncertain, however it can clinically resemble a number of conditions that carry more serious prognoses, such as tuberculosis (TB), toxoplasmosis, syphilis, lymphogranuloma venereum, SLE, and lymphoma [1,5]. In a case series by Fujimoto, 58\% of patients were misdiagnosed and inappropriately treated for lymph node TB before histopathology confirmed the diagnosis of KFD [5]. One report discussed a case of KFD with cutaneous manifestations that was initially diagnosed as a large cell lymphoma. The patient underwent one course of cytotoxic therapy before the correct diagnosis was made [1].

Diagnosis of KFD is made on lymph node biopsy that demonstrates paracortical foci of coagulative necrosis with karyorrhectic debris and a histiocytic cellular infiltrate $[1,3]$. The histopathologic finding of fragmented nuclear debris suggests apoptosis as the mechanism of cell death, and immunohistochemical findings, including increased Fas/FasL and CD 8+ T cells, further supports this mechanism [1]. Laboratory findings are typically normal, but elevated ESR and leukopenia can occur. Additionally, lab findings may show leukopenia, elevated ESR, and anemia. There is no effective treatment beyond symptom management with antipyretics, analgesics, and rest. However some reports have shown that patients with severe symptoms may benefit from corticosteroids [1].

\section{Conclusion}

Kikuchi-Fujimoto disease is rare, but awareness of it amongst clinicians is crucial when considering the differential diagnosis for a patient presenting with symptoms of fever and lymphadenopathy. KFD is a self-limited condition with an excellent prognosis, and management is typically supportive with rest and NSAIDs to alleviate symptoms. When it is mistaken for more severe disease, such as TB or malignant lymphoma, patients may be needlessly subjected to aggressive treatments.

\section{References}

1. Bosch X, Guilabert A, Miquel R, Campo E (2004) Enigmatic Kikuchi-Fujimoto disease: a comprehensive review. Am J Clin Pathol 122: 141-152. [Crossref]

2. Chiu CF, Chow KC, Lin TY, Tsai MH, Shih CM, et al. (2000) Virus infection in patients with histiocytic necrotizing lymphadenitis in Taiwan. Detection of Epstein-Barr virus, type I human T-cell lymphotropic virus, and parvovirus B19. Am J Clin Pathol 113: 774-781.[Crossref] 
3. Tsang WY, Chan JK, Ng CS (1994) Kikuchi's lymphadenitis. A morphologic analysis of 75 cases with special reference to unusual features. Am J Surg Pathol 18: 219-231. [Crossref]

4. Dorfman RF, Berry GJ (1988) Kikuchi’s histiocytic necrotizing lymphadenitis: an analysis of 108 cases with emphasis on differential diagnosis. Semin Diagn Pathol 5:329-345. [Crossref]

5. Aneja A, Maheswari K U1, H J GD1, Sheikh S2 (2014) A rare case of multifocal lymphadenopathy in a young male. Oxf Med Case Reports 2014: 141-144. [Crossref]

Copyright: (C2016 Lansky C. This is an open-access article distributed under the terms of the Creative Commons Attribution License, which permits unrestricted use, distribution, and reproduction in any medium, provided the original author and source are credited. 